regime until drug susceptibility studies are available, unless there is little possibility of primary resistance to INH.

Directly observed therapy (DOT) and HIV testing for all patients with TB are among the debated recommendations. In New York City alone, the cost of DOT is estimated to be $\$ 20$ million to $\$ 30$ million in one year. The HIV testing proposal calls for testing of all TB patients and those in close contact with them so that anyone infected with HIV can consider preventive treatment with INH.

In addition, the recommendations advise that anyone entering an isolation room should wear a disposable particulate respirator, another widely debated issue. Other recommendations include implementation of standard source control methods for negative pressure isolation rooms with exhaust air not being recirculated to other rooms or outside to sites near air intakes. Supplemental approaches for germicidal ultraviolet irradiation and high-efficiency particulate air (HEPA) filtration and strategies for recognizing and managing non-compliant behavior are also included.

These joint TB control recommendations provide guidance for a wide variety of populations and settings, including state, city, and county TB control programs; other health department or hospital outpatient programs, such as refugee programs, sexually transmitted disease clinics, HIV clinics; acute-care and extended-care facilities; substance abuse treatment programs; shelters for the homeless; day-care centers; and other institutions.

FROM: Control of tuberculosis in the United States. Am Rev Respir Dis. 1992;146:1623-33.

\section{New AIDS Definition Effective 1993}

Since 1981, about 250,000 individuals have been diagnosed as having AIDS and 160,000 have died. On January 1, 1993, a new definition of AIDS went into effect, resulting in thousands more individuals with the diagnosis of AIDS. The new definition adds diseases that are more common to women and intravenous drug users, including pulmonary tuberculosis (TB), recurrent pneumonia, and invasive cervical cancer.

Under the new definition, 90,000 more Americans are expected to be diagnosed as having AIDS this year, compared with an average of 50,090 under the old definition. The result will be a dramatic increase in demand for the already overwhelmed treatment and social services available.

One of the most important new indicators is those individuals with CD4 cell counts under 200. An estimated 1 million Americans are infected with HIV, and up to 190,000 have CD4 counts less than 200. Experts believe that many of these individuals do not know they are infected with HIV.

CDC epidemiologists hope the new definition will draw attention to the importance of HIV testing and focus attention on the need for doctors to consider co-infection in patients with TB and cervical cancer who have never been tested.

\section{Unions Ask OSHA for TB Enforcement Guidelines}

A coalition of 11 unions and the AFL-CIO asked the Department of Labor at the end of 1992 to issue tuberculosis enforcement of strict TB controls in healthcare facilities and other workplaces in the country. The coalition view is that a permanent standard will be needed eventually to protect workers against TB.

The coalition also called for immediate release of a "Joint Advisory Notice" from OSHA and the Department of Health and Human Services (CDC) to all employers to provide guidance on protecting workers from occupational TB exposure.

Enforcement guidelines are already in place in some parts of the country. Federal OSHA's Region 2 (NY, NJ, PR, VI) issued enforcement guidelines for TB control in May 1992, which have been the subject of debate because certain recommendations go beyond the CDC's current December 1990 "Guidelines for Preventing Transmission of Tuberculosis in Healthcare Settings." On December 1, 1992, California OSHA issued interim TB control enforcement guidelines and has announced that there will be programmed inspections of high-risk work environments for $\mathrm{TB}$, including extended-cam facilities, hospitals, and prisons.

The CDC invited healthcare consultants and union representatives to a meeting on January 23, 1993, to review and discuss a revised draft of the CDC's December 1990 TB guidelines. The draft had more specific information on patient and healthcare worker education, the immunocompromised healthcare worker, and ventilation, including ultraviolet germicidal irradiation and HEPA filtration. Opinions from consultants differed greatly on the most appropriate type of respiratory protection.

\section{Offering Influenza Vaccination to House Staff During Medical Conferences Achieves Compliance}

Ninety percent of residents and junior medical students offered influenza vaccine were immunized during attendance at conferences and in clinics, com- 